\title{
Alternative Method for the Metrological Characterization of Spur Gears in the Sub- Millimeter Range Using Optical Equipment
}

\author{
J. Caja, E. Gómez, C. Barajas, P. Maresca, M. Berzal \\ Departamento de Mecánica Industrial - Universidad Politécnica de Madrid \\ Ronda de Valencia, 3 - 28012 Madrid - España \\ jesus.caja@upm.es
}

\begin{abstract}
The aim of this work is to develop a software that allows the inspection of spur gear manufactured in the sub-millimeter range. The measurements are made using a digital optical machine and using an analysis proprietary software implemented in Matlab ${ }^{\circledR}$, which is able to handle images, captured using the digital optical machine. The software allows to evaluate the profile and pitch deviations as establish in the ISO/TR 10064-1:1992 standard [1].
\end{abstract}

Keywords: Metrology, Optical Measuring Machine, Gear Measurement, Spur Ger

PACS: 06.20.-f, 06.30.Bp, 06.60.Mr, 89.20.Bb, 89.20.Ff, 89.20.Kk

\section{INTRODUCTION}

Systems for power transmission based on gears are one of the most widely used today in fields such as automotive, aerospace, machine tools and other applications [2]. Cause of this so extensive use, greater demands in terms of transmitted power, durability, life... are required. Also, a reduction of their production costs, noise, etc. This situation has led to the tolerances allowed for these elements become increasingly narrow, forcing more precise manufacturing processes, being of vital importance the dimensional verification.

The verification of the gears is a costly process, and that is currently carried out by using three Coordinates Measuring Machine (CMM) or specific machines for measuring gear, equipped with numerical control systems. The use of this equipment reduces the measurement time and the associated uncertainties. Measured phases with this equipment can be summarized as: alignment of the workpiece, the scanning surface, data evaluation and presentation of results.

The development of micro-manufacturing techniques has allowed the manufacture of spur gears in the sub-millimeter range. In this case, the use of traditional system of gear check, described above, is unfeasible due to the impossibility of probing on the surfaces to be evaluated. In this assumption the special optical equipment and optical digital machines are a viable alternative. The use of these equipments has, among others, the following advantages: no probing, speed of data acquisition (taking a series 
of images) and no need to align the workpiece to be evaluated. Also, we can also use this technique to evaluate this type of gear (straight) in any dimension.

From an analytical point of view, optical equipment can be considered a three measuring coordinates that without $Z$ axis. This assumption implies that the plane on which to perform the measurement of the workpiece is parallel to the XY plane of the machine, restriction that limits the type of workpiece to characterize.

Among the parameters to be evaluated according to the technical recommendation ISO/TR 10064-1:1992 [1] are: pitch deviations (Figure 1), single and cumulative, and the evaluation of the profile deviations of the gear tooth (Figure 4).

\section{EVALUATION OF THE PITCHES DEVIATIONS}

In gear metrology, the pitch and deviations from it are always arcs of a circle. Depending on the considered radius (base radius, reference radius, tip radius, root radius,...) different values for the pitch and its deviations are defined. Among these, the measured pitch on the reference circle $\left(p_{t}\right)$ and the one measured on the base circle $\left(p_{b}\right)$ are most relevant. The pitch pt is defined as the length of the arc measured on the reference circle between two consecutive flanks (right or left) of the tooth profile (Figure 1).

$$
\begin{aligned}
& p_{t}=\pi \cdot m=r_{p} \cdot \theta \\
& \theta=\frac{2 \cdot \pi}{z}
\end{aligned}
$$

Also, the base pitch is defined as the length of the arc measured on the base circle (right or left) between two consecutive flanks of the tooth profile.

$$
\begin{aligned}
& p_{b}=\pi \cdot m=r_{b} \cdot \theta \\
& \theta=\frac{2 \cdot \pi}{z}
\end{aligned}
$$

Where $m$ is the module of the gear, $z$ is the number of teeth, $r_{p}$ is the reference radius of the gear defined as $z \cdot m, r_{b}$ is the base radius calculated as $r_{p} \cdot \cos \alpha$ and $\alpha$ is the pressure angle of gear.

Determination of the pitch deviations is one of the most important checks to realize. We can distinguish different types of deviations: single deviations between two adjacent flanks $\left(f_{p t}\right)$ and deviations pitch accumulated on a specific number of pitches $\left(F_{p k}\right)$, among others (Figure 1). Traditional methods, based on instruments that do the probing of the surface, present two major problems: the need of a rotary table and accurate determination of the axis rotation of gear (made by different methods). 


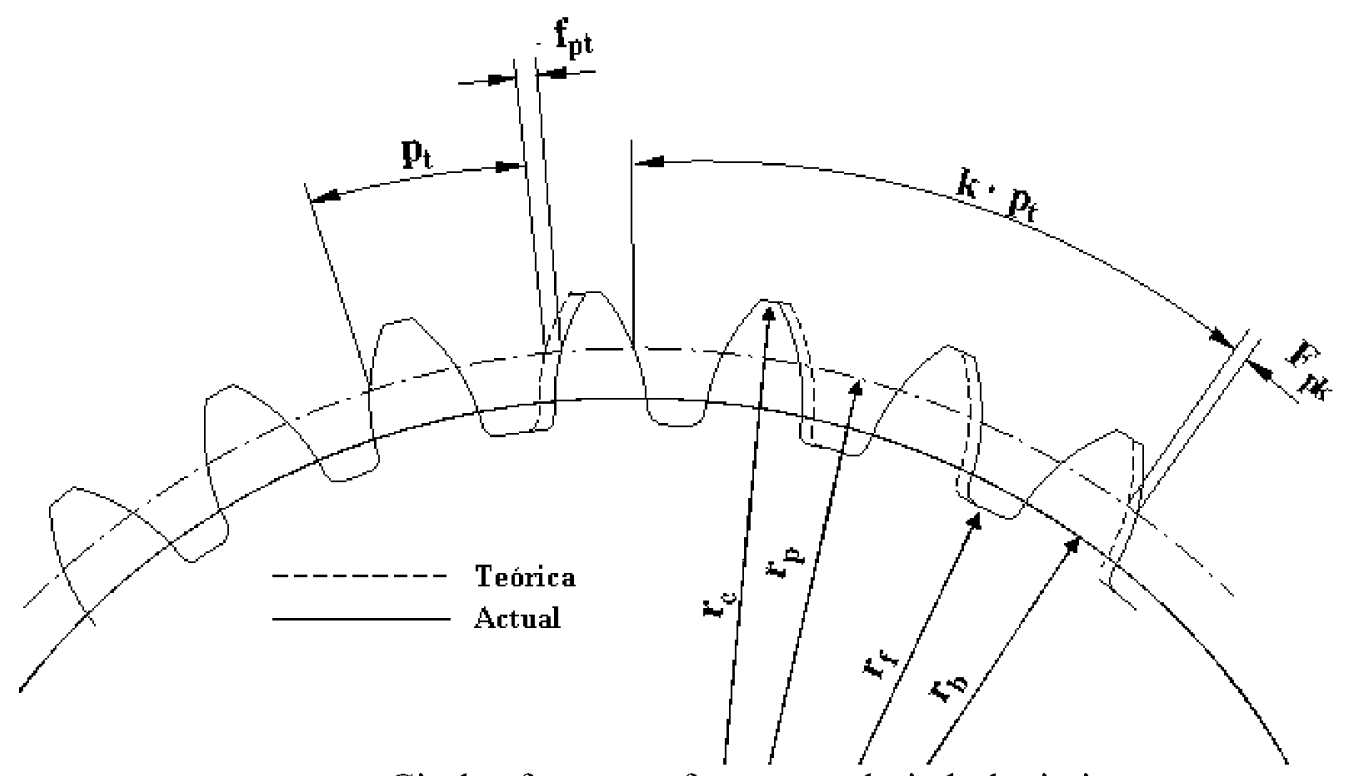

FIGURE 1. Circles features of a gear and pitch deviations.

\section{EVALUATION OF THE INVOLUTE PROFILE}

For the evaluation of the involute profile, we have started from the work of Shumungan [3]. Figure 2 shows an involute curve generated from point $\mathrm{C}$ to $\mathrm{P}$ from the tangent line at this point, rotated of an angle $\theta$. The family of tangent lines that surrounds the base circle can be expressed parametrically as:

$$
x \cdot \cos \theta+y \cdot \sin \theta-r_{b}=0
$$

where $x$ and $y$ represent the coordinates of a point belonging to the involute curve and $r_{b}$ is the radius of the base circle.

Likewise, and seeing the Figure 2, the length of the segment PT must be equal to the length of the arc TC. This relationship can be expressed analytically as:

$$
\left(x-r_{b} \cdot \cos \theta\right)^{2}+\left(y-r_{b} \cdot \sin \theta\right)^{2}=\left(r_{b} \cdot \theta\right)^{2}
$$

Relating equations 3 and 4 , the coordinates $x, y$ of a point belonging to the involute profile can be expressed as [4]:

$$
\begin{aligned}
& x=r_{b} \cdot \cos \theta+r_{b} \cdot \theta \cdot \sin \theta \\
& y=r_{b} \cdot \sin \theta-r_{b} \cdot \theta \cdot \cos \theta
\end{aligned}
$$

By varying the rotation angle $\theta$ from 0 to a suitable value, a series of points, that describe the involute profile so that $r=\sqrt{x^{2}+y^{2}}$ range between base circle and tip circle $r_{c}$, are obtained. 


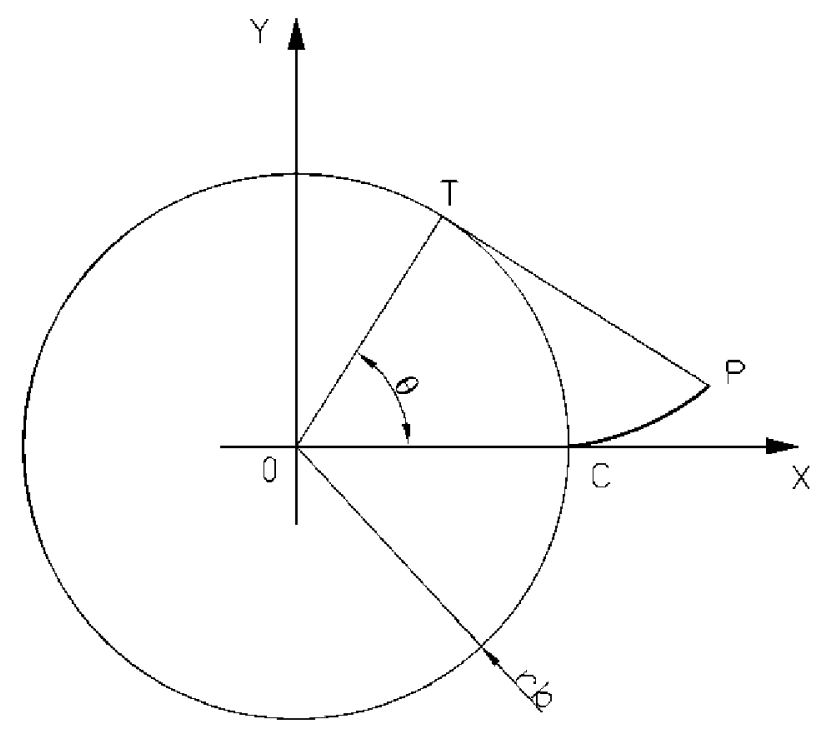

FIGURE 2. Involute curve.

\section{Verification of the involute profile deviations}

According to the standard ISO 1328-1:1995 [5], the involute profile deviations correspond to the normal distances corresponding between a point on the gear tooth profile and the one corresponding to theoretical profile of the involute curve. Moreover, these deviations are measured in planes transverse to the tooth.

The calculation model used to determine these deviations will be the following:

According to Figure 3, the $\mathrm{x}$-axis must pass through point $\mathrm{C}$, base of the involute curve. Suppose that the point $\mathrm{M}$ of coordinates $\left(x_{m}, y_{m}\right)$ corresponds to a point on the gear tooth profile, the tangent line to the base circle that passes through this point can be obtained by the following equation:

$$
x_{m} \cdot \cos \theta_{m}+y_{m} \cdot \sin \theta_{m}-r_{b}=0
$$

where the only unknown value is the rotation angle $\theta_{\mathrm{m}}$. Mathematical operator, this can be obtained as:

$$
\cos \theta_{m}=\frac{r_{b} \cdot x_{m}-\sqrt{r_{b}^{2} \cdot x_{m}^{2}+\left(x_{m}^{2}+y_{m}^{2}\right)\left(y_{m}^{2}-r_{b}^{2}\right)}}{\left(x_{m}^{2}+y_{m}^{2}\right)}
$$

The normal deviation between the measured point and the theoretical point, seeing Figure 3, can be expressed as: 


$$
\begin{aligned}
& \Delta_{m}=\mathrm{PM}=\mathrm{P}^{\prime} \mathrm{M}^{\prime}=\mathrm{T}^{\prime} \mathrm{M}^{\prime}-\mathrm{T}^{\prime} \mathrm{P}^{\prime} \\
& \Delta_{m}=\sqrt{\left(x_{m}-r_{b} \cdot \cos \theta_{m}\right)^{2}+\left(y_{m}-r_{b} \cdot \sin \theta_{m}\right)^{2}}-r_{b} \cdot \theta_{m}
\end{aligned}
$$

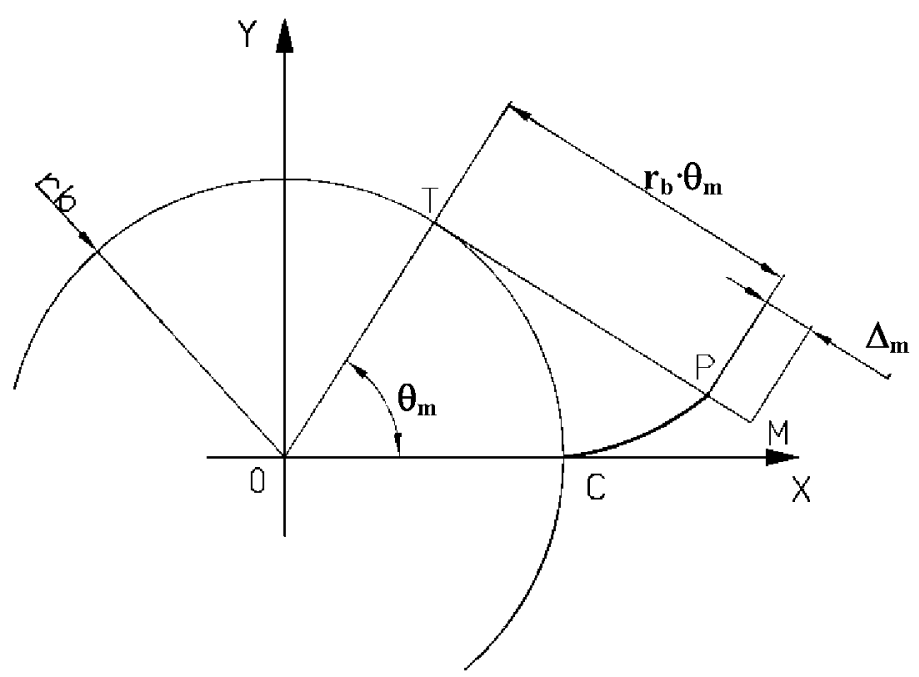

FIGURE 3. Deviations from the theoretical involute profile

These deviations are represented in the typical diagram of the profile (Figure 4). Deviations of the curve respect to a straight line represent the deviations of the profile about involute curve generated from the base circle of the gear inspected.

Starting from the tip of the tooth (point A, Figure 4), the theoretical profile contrasts with the measured profile in the range $L_{A E}$ (including the evaluation length $L_{\alpha c}$ more the tip area of the tooth). The distance between the highest and lowest profile, measured by a horizontal line, represents the total profile deviation $F_{\alpha}$. Midline of the profile (dashed line) is determined and two lines parallel to this, containing all points of the profile, are established. Distance measured according to a horizontal line between these two lines is the shape deviation of the profile $f_{f o} . F_{H \alpha}$ represents the deviation of the profile (distance between a vertical line and the midline of the profile measured to the distance $L_{\alpha c}$ ). 


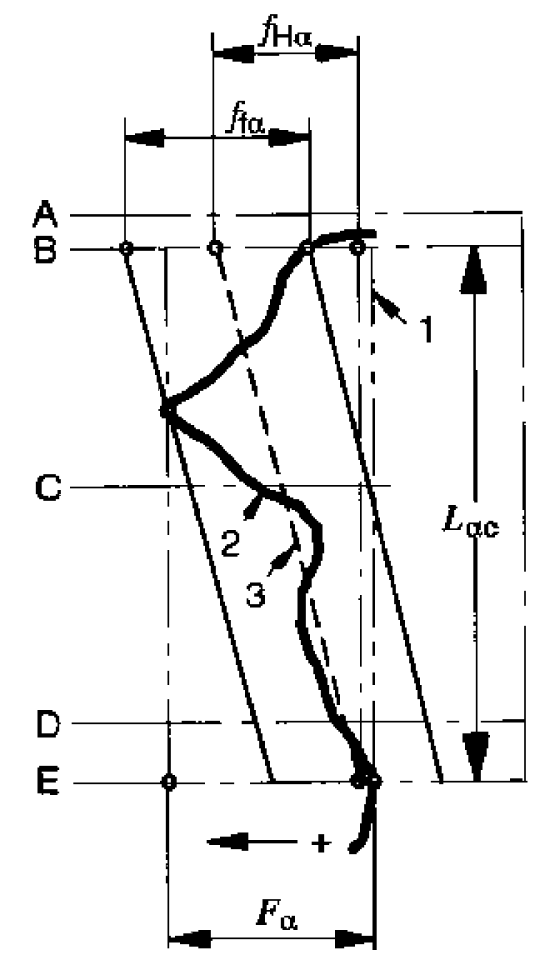

FIGURE 4. Diagram of the involute profile deviations of a gear tooth

\section{IMPLEMENTATION OF COMPUTATIONAL ALGORITHMS}

The developed algorithms allow to (a) get the edge of the gear from the digital image obtained by the CCD camera, (b) identifying pixels belonging to it and (c) determine the results of the various checks described above.

The made measurements allow to vary the used magnification of the optical equipment and the number of pixels supposed on the profile. The developed algorithms have been implemented in MatLab R2009b, together effective library for image analysis, Toolbox Image Processing. From the work of Midoh et al $[6,7]$, the edge detection technique based on the Canny filter, which for metrological images obtained for workpieces of sub-millimeter range is the most effective in terms of probability, uncertainty and precision [8], has been decided to use. Also, to determine more accurate edge, techniques subpixel and thresholding have been used [9]. Stages of the measurement process for previous checks are indicated and illustrated then. As initial image obtained by a digital optical machine Tesa Visio 300, from a gear module 1 and 32 teeth, has been used. One of the problems of the edge determination is to differentiate whether a potential outlier is part of a manufacturing defect or is a foreign particle (dust or dirt). For an user is a simple task, but it is certainly difficult to implement automatically. 


\section{Case 1: Evaluation of the pitch deviations}

Step 1: The algorithm receives as inputs: (a) the image to be treated (Figure 5), (b) the coordinates of pixels in the upper left and lower right corners, and the module and the number of gear teeth.

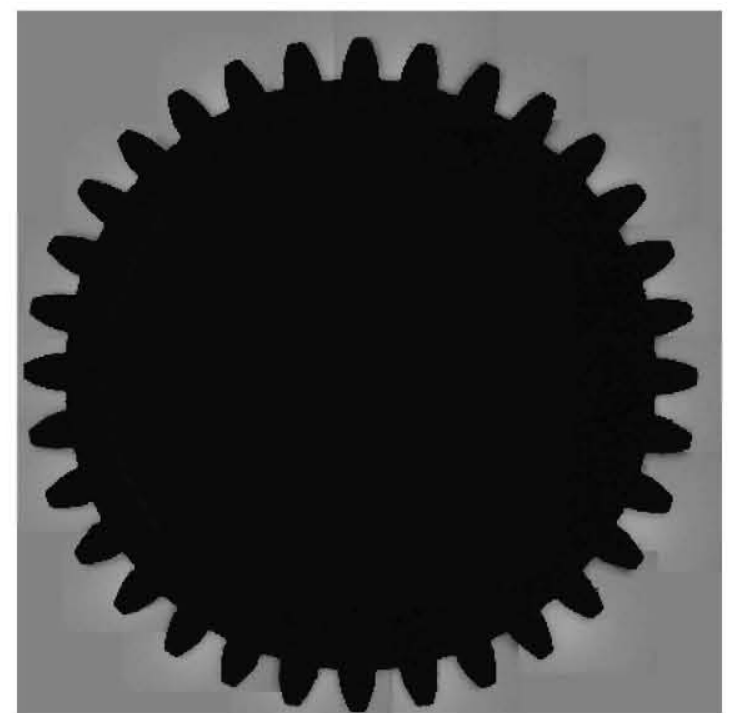

FIGURE 5. Initial Screen gear, module 1 and 32 teeth. (Area $36.5 \times 36.5 \mathrm{~mm}$ )

Step2: To perform optimally edge detection, this is done from circles defined by the user on the image captured by the optical machine (in green color showed in Figure 6). Then, the algorithm establishes rectangular areas of evaluation (Figure 6) to which the Canny filter and the threshold value are applied [10]. Finally, the algorithm selects the edge pixels and determines their coordinates (Figure 7).

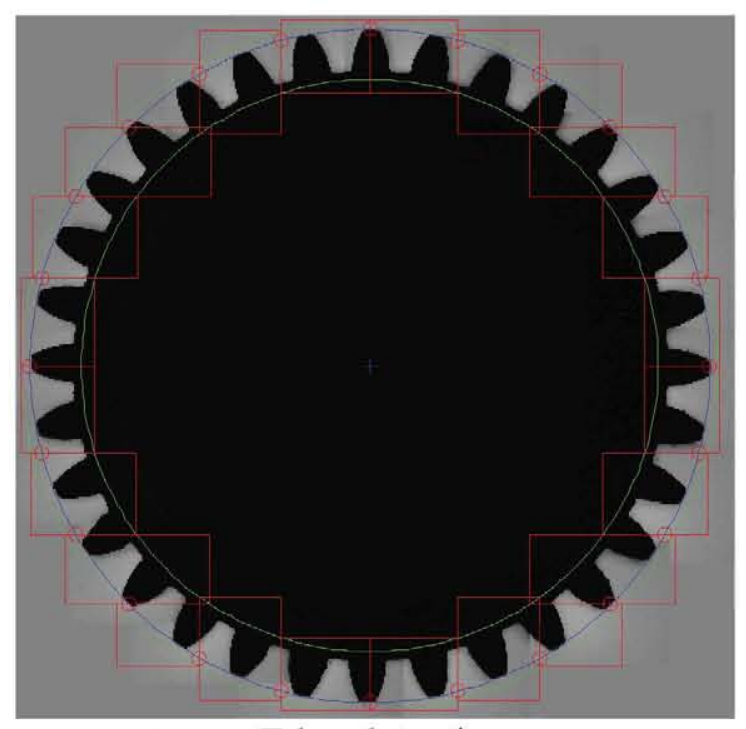

FIGURE 6. Edge detection zones. 
Step 3: With these coordinates, and a computation iterative process, the algorithm, based on the coordinates of pixels, determines the position of the rotation center of the gear. From this center, feature circles of the gear are established (Figure 7).

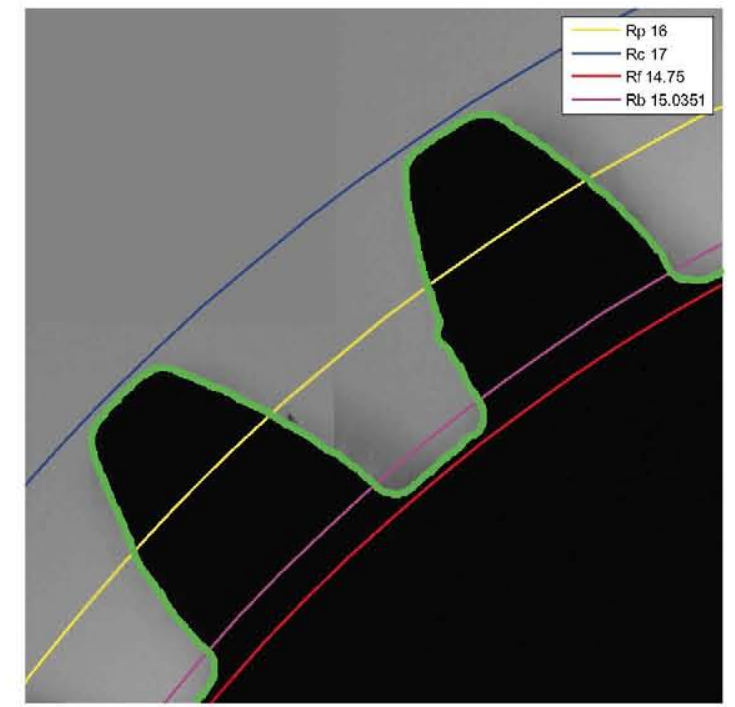

FIGURE 7. Detected edge and feature circles

Step 4: From center established in the previous step, the points, located on the flanks of the teeth, that have a distance to the rotation center of the gear equal to feature circle one in which we want to evaluate the pitch deviations, are determined. A detection interval is stabled due to the difficulty of finding a point on the feature circle.

Step 5: Finally, and from the above points detected, taking into account equations 1 and/or 2, pitch deviations are calculated. Figure 8 shows some of the results that the program provides.

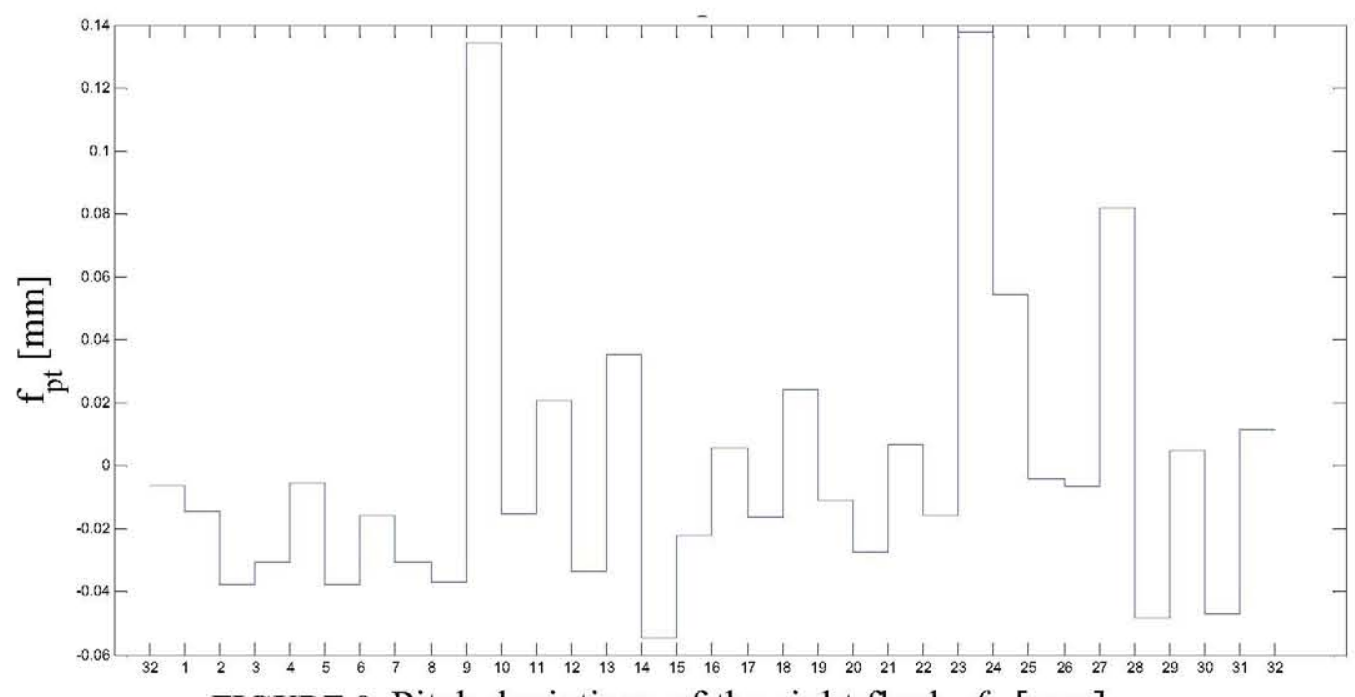

FIGURE 8. Pitch deviations of the right flank, $f_{p t}[\mathrm{~mm}]$ 


\section{Case 2: Evaluation of the profile deviations}

Step 1: The algorithm receives as inputs: (a) the image to be treated (Figure 9), (b) the coordinates of pixels in the upper left and lower right corners, and information about the rotation center of the gear and feature circles, predetermined.

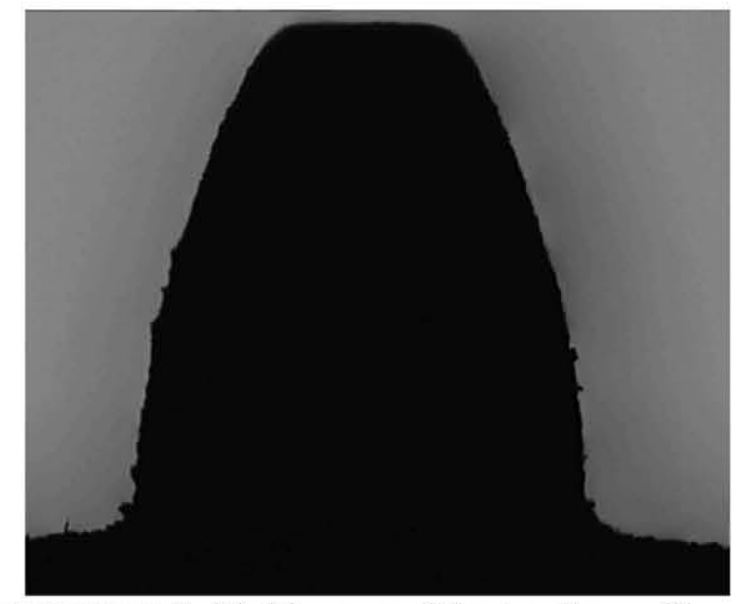

FIGURE 9. Initial image of the tooth profile

Step2: The edge detection is made, similar to the one form explained in paragraph 4.1-Step 2, except that the study area, defined by the user, is rectangular. The pixels of the edge and their coordinates are obtained.

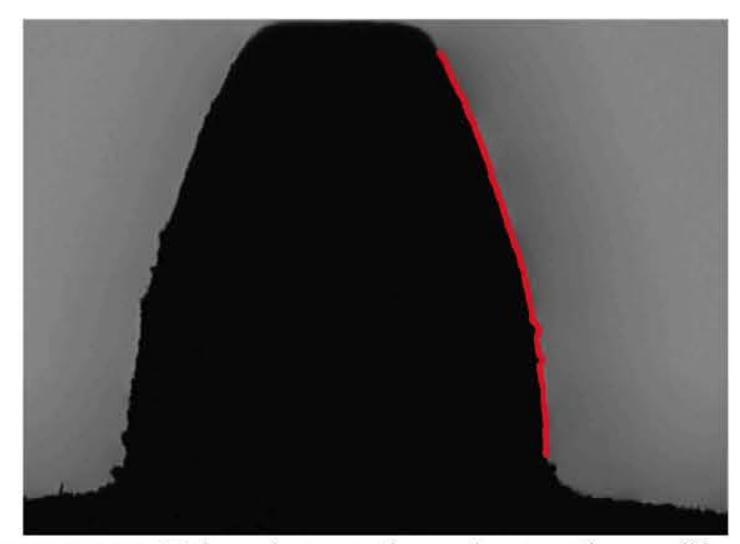

FIGURE 10. Edge detected on the tooth profile.

Step 3: The algorithm separates the pixels on the edge belonging to the right and left flank. Also, we determine that the pixels of the edge are on the active area of the profile, between the base circle and the head, lowered a certain value (Figure 10). After realizing a change of coordinates system to use the equations of the involute profile (eq. 5), the theoretical profile (blue points) against the measured profile (red points) are showed in Figure 11. 


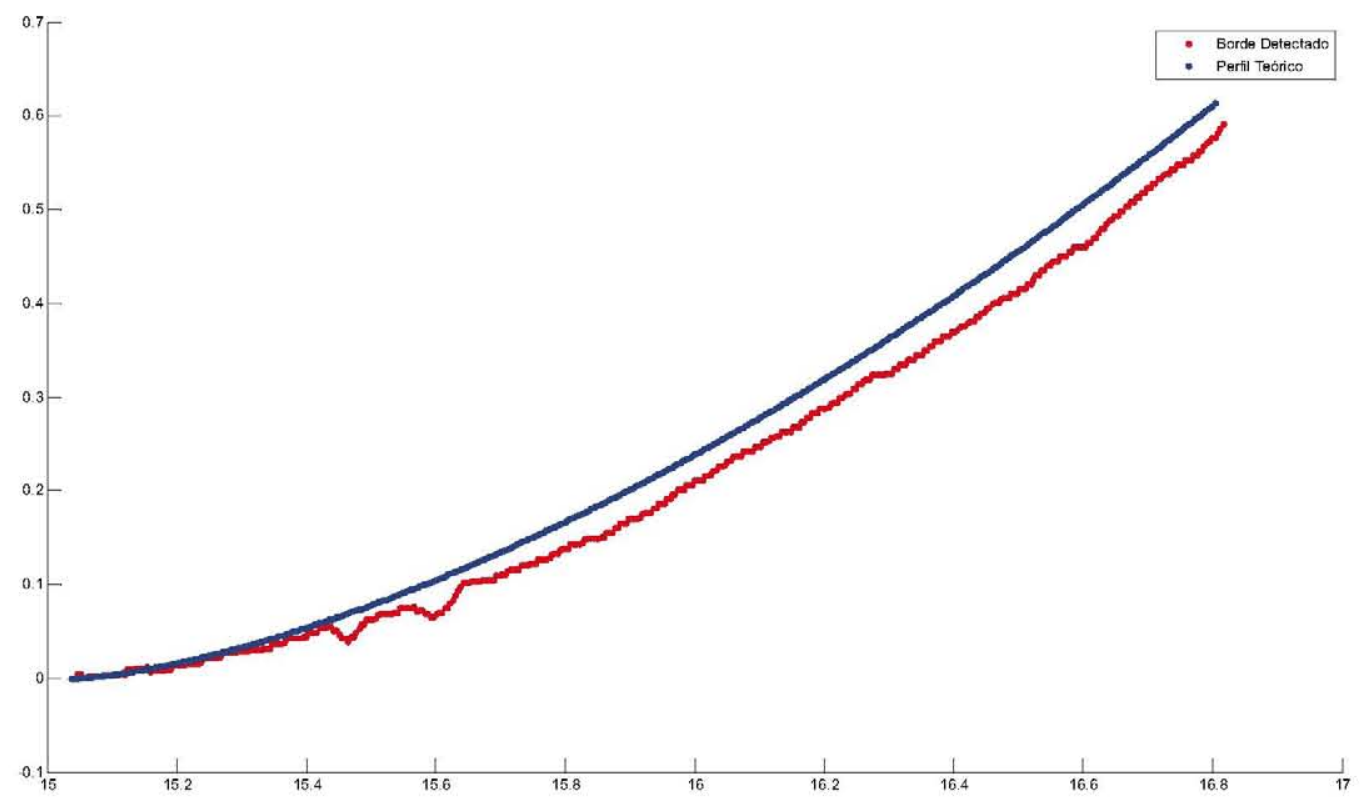

FIGURE 11. Measured profile versus theoretical profile

Step 4: Finally, the normal deviations between the theoretical profile and the measured profile (eq. 8) are obtained. The Figure 12 shows the typical representation of these deviations.

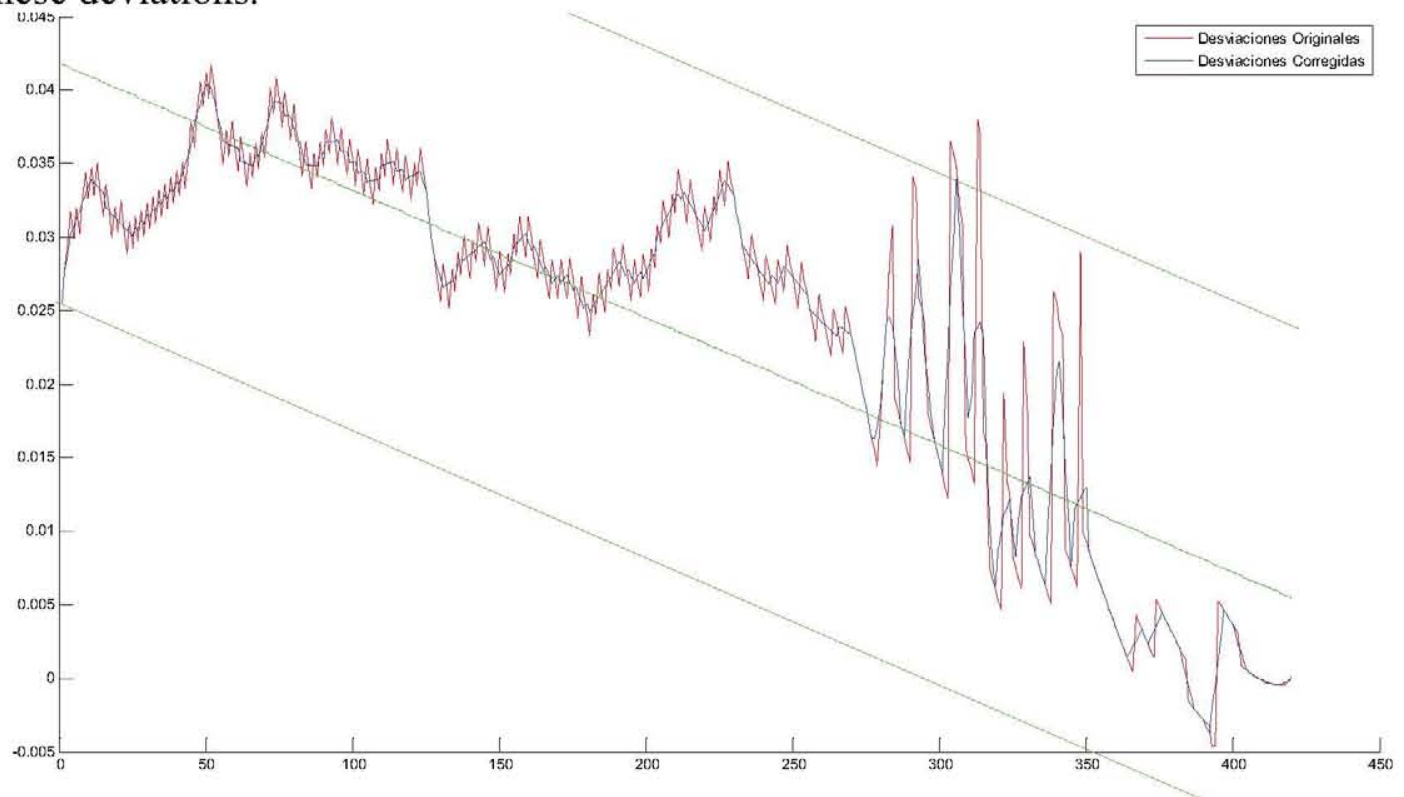

FIGURE 12. Diagram of deviations of the involute profile 


\section{ESTIMATING UNCERTAINTY}

The use of the Monte Carlo method to obtain the uncertainty of the parameters previously calculated has been used. We have followed the recommendation of the Supplement 1 to the 'ISO Guide to the Expression of Uncertainty in Measurement' [11]. The considered components of the uncertainty are:

Associated to the coordinates of the pixels lying at the edge. The digital optical machine has been calibrated, so that the traceability of the above coordinates is ensured. This coordinates, are sampled from a normal distribution with the mean value equal to the coordinate one and the standard deviation equal to the standard uncertainty obtained in the calibration of the machine

Following the recommendations in section 7.2.1 of the Supplement 1 to the 'ISO Guide to the Expression of Uncertainty in Measurement' [11], the model is replicated $\mathrm{N}=10^{6}$ times, establishing a coverage interval for an approximate probability of $95 \%$.

The results obtained in different simulations and with different magnifications (high values of magnification give lower value of pixel size) show that the expanded uncertainty of the measurements takes values between 2 or 3 times the value of the pixel size.

\section{CONCLUSIONS}

The algorithms developed in this study, have allowed to evaluate the geometric parameters, in particular the pitch and the involute profile of the gears teeth, the pitch, and the involute profile of the teeth, from a digital image obtained with a digital optical machine. These algorithms have been proven robust in use during the verification of gear of sub-millimeter dimensions.

The use of optical equipment allows verify gear that otherwise would not be possible, with a number of advantages such as:

- Process automation

- High speed of the verification process (only is necessary to determine a number of images of the gear, function of the used magnification)

- Measurements with an associated uncertainty of about 2 to 3 times the instrument resolution.

- Elimination of problems such as the need to use rotary table and accurate determination of the rotation axis of the gear.

- The use of the developed algorithms can be extended to larger gears, obtaining the same advantages as described above. 


\section{REFERENCES}

1. ISO, ISO/TR 10064-1:1992 Cylindrical gears - Code of inspection practice - Part 1: Inspection of corresponding flanks of gear teeth, 1992

2. G. Goch, Manufacturing Technology 52 (2003).

3. M.S. Shumungan, S.V.R. Surya and V. Jayaprakash, Mech. Mach. Theory 33 (1998).

4. F. L. Litvin and A. Fuentes, Gear Geometry and applied theory 2nd Ed, Cambridge:Cambridge University Press, 2004.

5. ISO, ISO/TR 1328-1:1995 Cylindrical gears - ISO systems of accuracy - Part 1: Definitions and allowable values of deviations relevant to corresponding flanks of gear, 1995

6. Y. Midoh, K. Nakamae and H. Fujioka, Meas. Sci. Technol 18 (2007).

7. Y. Midoh, K. Miura, K. Nakamae and H. Fujioka, Meas. Sci. Technol 16 (2005).

8. G. Iannizzotto and L. Vita, "A multiscale turning angle representation of object shapes for image retrieval" in Visual 99, Amsterdam, (The Netherlands), 1999.

9. R. C. Gonzalez, R. E. Woods and S. L. Eddins, Digital Image Processing Using MATLAB 2nd Ed, Knoxville:Gatesmark Publishing, 2009.

11. P. Maresca, E. Gómez, J. Caja, C. Barajas and M. Berzal, Measurement, 43 (2010).

12. BIPM, JCGM 101:2008. Evaluation of measurement data - Supplement 1 to the "Guide to the expression of uncertainty in measurement" - Propagation of distributions using a Monte Carlo method, 2008, URL: http://www.bipm.org/en/publications/guides/gum.html 